\title{
Adjuvant Potential of Selegiline in Attenuating Organ Dysfunction in Septic Rats with Peritonitis
}

\author{
Cheng-Ming Tsao ${ }^{1,2}$, Jhih-Gang Jhang ${ }^{3}$, Shiu-Jen $\mathrm{Chen}^{4,5}$, Shuk-Man $\mathrm{Ka}^{6}$, Tao-Cheng $\mathrm{Wu}^{7,8}$, Wen- \\ Jinn Liaw ${ }^{2,9}$, Hsieh-Chou Huang ${ }^{10 * 9}$, Chin-Chen $\mathrm{Wu}^{3,11 * 9}$
}

1 Department of Anesthesiology, Taipei Veterans General Hospital, and National Yang-Ming University, Taipei, Taiwan, R.O.C., 2 Department of Anesthesiology, Tri-Service General Hospital, National Defense Medical Center, Taipei, Taiwan, R.O.C., 3 Department of Pharmacology, National Defense Medical Center, Taipei, Taiwan, R.O.C., 4 Department of Nursing, Kang-Ning Junior College of Medical Care and Management, Taipei, Taiwan, R.O.C., 5 Department of Physiology, National Defense Medical Center, Taipei, Taiwan, R.O.C., 6 Graduate Institute of Aerospace and Undersea Medicine, National Defense Medical Center, Taipei, Taiwan, R.O.C., 7 Division of Cardiology, Department of Medicine, Taipei Veterans General Hospital, Taipei, Taiwan, R.O.C., 8 Cardiovascular Research Center, National Yang-Ming University, Taipei, Taiwan, R.O.C., 9 Department of Anesthesiology, Tungs' Taichung MetroHarbor Hospital, Taichung, Taiwan, R.O.C., 10 Department of Anesthesiology, Cheng-Hsin General Hospital, Taipei, Taiwan, R.O.C., 11 Department of Pharmacology, Taipei Medical University, Taipei, Taiwan, R.O.C.

\begin{abstract}
Selegiline, an anti-Parkinson drug, has antioxidant and anti-apoptotic effects. To explore the effect of selegiline on sepsis, we used a clinically relevant animal model of polymicrobial sepsis. Cecal ligation and puncture (CLP) or sham operation was performed in male rats under anesthesia. Three hours after surgery, animals were randomized to receive intravenously selegiline $(3 \mathrm{mg} / \mathrm{kg})$ or an equivalent volume of saline. The administration of CLP rats with selegiline (i) increased arterial blood pressure and vascular responsiveness to norepinephrine, (ii) reduced plasma liver and kidney dysfunction, (iii) attenuated metabolic acidosis, (iv) decreased neutrophil infiltration in liver and lung, and (v) improved survival rate (from $44 \%$ to $65 \%$ ), compared to those in the CLP alone rats. The CLP-induced increases of plasma interleukin-6, organ superoxide levels, and liver inducible nitric oxide synthase and caspase-3 expressions were ameliorated by selegiline treatment. In addition, the histological changes in liver and lung were significantly attenuated in the selegiline -treated CLP group compared to those in the CLP group. The improvement of organ dysfunction and survival through reducing inflammation, oxidative stress and apoptosis in peritonitis-induced sepsis by selegiline has potential as an adjuvant agent for critical ill.
\end{abstract}

Citation: Tsao C-M, Jhang J-G, Chen S-J, Ka S-M, Wu T-C, et al. (2014) Adjuvant Potential of Selegiline in Attenuating Organ Dysfunction in Septic Rats with Peritonitis. PLoS ONE 9(9): e108455. doi:10.1371/journal.pone.0108455

Editor: Cordula M. Stover, University of Leicester, United Kingdom

Received May 16, 2014; Accepted August 29, 2014; Published September 30, 2014

Copyright: (C) 2014 Tsao et al. This is an open-access article distributed under the terms of the Creative Commons Attribution License, which permits unrestricted use, distribution, and reproduction in any medium, provided the original author and source are credited.

Data Availability: The authors confirm that all data underlying the findings are fully available without restriction. All relevant data are within the paper and its Supporting Information files.

Funding: This work supported by grants from National Science Council, Taiwan, R.O.C. (NSC 97-2320-B-016-006-MY3, 98-2314-B-075-005-MY3 and 100-2320-B016-008) and Cheng Hsin General Hospital, Taiwan, R.O.C. (CHGH 99-61). The funders had no role in study design, data collection and analysis, decision to publish, or preparation of the manuscript.

Competing Interests: The authors have declared that no competing interests exist.

* Email: hc1.huang@xuite.net (HCH); ccwu@office365.ndmctsgh.edu.tw (CCW)

9 These authors contributed equally to this work.

\section{Introduction}

Despite advances in critical care medicine, sepsis continues to be a serious clinical entity with mortality rate still $30-50 \%$ for severe sepsis [1]. Numerous clinical trials of cytokine-specific therapies failed to improve survival in patients with sepsis, however recently, using pharmacological modulators to suppress apoptosis has been shown a striking efficacy in animal models of sepsis [2-5].

Selegiline (SEL, L-deprenyl), a monoamine oxidase-B (MAO-B) inhibitor, is a useful anti-Parkinson drug both in monotherapy and as an adjunct to levodopa therapy [6-8]. The MAO-B inhibitor could protect neuronal cells by its antioxidant and anti-apoptotic effects $[9,10]$. The neuroprotection effects of SEL in laboratory models may be associated with the decrease of oxidative stress, stabilization of mitochondria a)nd prevention of pro-apoptotic signaling process [11,12]. In addition to the treatment of neurodegenerative disorder, SEL reduces brain damage and enhances recovery after stroke in rats and humans [13-15]. Moreover, SEL increases free radical elimination and apoptosis suppression in aged liver and collapsing heart [16,17]. SEL has been also shown to reduce vascular permeability and lung injury in a rodent hemorrhagic shock model, mostly due its anti-apoptotic action [18]. However, no studies have shown the impact of SEL at attenuating organ dysfunction and increasing survival in sepsis. In the current study, we have tested, using a rat model of cecal ligation and puncture (CLP)-induced sepsis, the hypothesis that SEL improved survival in an intra-abdominal sepsis via its antioxidant and anti-apoptotic effects.

\section{Materials and Methods}

\section{Rat model of sepsis}

Seventy-two male Wistar rats (280-350 g) were used in this study. All work was approved by the Committee on the Ethics of Animal Experiments of Cheng-Hsin General Hospital (Permit Number: CHGH 99-61), and the care and handling of the animals were in adherence to the National Institutes of Health Guidelines 
for ethical animal treatment. Rats were bred and maintained under a 12-h light/dark cycle at a controlled temperature $\left(21 \pm 2^{\circ} \mathrm{C}\right)$ with free access to food and tap water.

\section{Surgical procedures}

Catheter placements of left carotid artery and right jugular vein were performed for blood pressure measuring and drugs administering, respectively. The catheters were cannulated and exteriorized to the back of the neck under anesthesia of intraperitoneal sodium pentobarbital $(40-50 \mathrm{mg} / \mathrm{kg})$ and inhalational isoflurane $(0.5 \%-1 \%)$ given via nosecone. After surgery, the cannulated animals were allowed to recover to the normal condition overnight with standardized pellet food and tap water ad libitum.

The intraperitoneal sepsis was induced by CLP using methods described previously [19]. Briefly, a midline laparotomy was performed under anesthesia of intravenous pentobarbital and inhalational isoflurane. The exposed cecum was ligated with a 3-0 silk ligature just distal to the ileocecal valve, punctured twice at opposite ends with an 18-gauge needle. The cecum was replaced into the abdominal cavity and the abdominal incision was closed. In addition, $0.2 \%$ lidocaine was used to infiltrate surgical wound in each rat after incision closure for post-operative analgesia. The rats in sham control were performed laparotomy and cecal exposure without any other manipulation. All animals immediately received normal saline solution $(20 \mathrm{~mL} / \mathrm{kg}$ subcutaneous) after operation.

\section{Experimental design}

Animals were divided into four groups: (1) sham operation (SOP) group: $0.9 \%$ saline ( $3 \mathrm{~mL} / \mathrm{kg}$ intravenous for $10 \mathrm{~min}$ ) at $3 \mathrm{~h}$ after sham operation, (2) SOP + SEL: SEL (3 mg/ $\mathrm{kg}$ intravenous for $10 \mathrm{~min}$ ) at $3 \mathrm{~h}$ after sham operation, (3) CLP group: same regimen of saline at $3 \mathrm{~h}$ after CLP surgery, and (4) CLP + SEL group: same regiment of SEL at $3 \mathrm{~h}$ after CLP surgery. The dose of SEL $3 \mathrm{mg} / \mathrm{kg}$ used in this study was based on our preliminary data showing that it was effective on reducing the superoxide level raised by CLP surgery in liver and lung, while $1 \mathrm{mg} / \mathrm{kg}$ of SEL did not improve the survival of CLP rats. SEL (Sigma-Aldrich, St. Louis, MO, USA) was dissolved in $0.9 \%$ saline at the concentration of $1 \mathrm{mg} / \mathrm{mL}$.

All rats enrolled in the study were kept in the small in-house animal facility of our institute to enable optimal monitoring: the overall health status was checked every $2-3 \mathrm{~h}$ for signs of distress. As suggested by Nemzek et al. [20], we followed a more accurate, empirically established set of guidelines that offered, although a very narrow, yet a feasible window of opportunity for induction of death. Specifically, rats were euthanized only at the end of each experiment (at $18 \mathrm{~h}$ after CLP or sham surgery) or upon signs of imminent death (i.e. unresponsive to external stimuli, inability to maintain upright position/tremor and prolonged/deep hypothermia and/or agonal breathing) by using an overdose of pentobarbital $(100 \mathrm{mg} / \mathrm{kg}$, i.v.). Then, some tissue specimens of liver and lung were immediately exercised to analyze superoxide levels, Western blotting and histological changes. In addition, the survival rate at $18 \mathrm{~h}$ in each group was analyzed.

\section{Measurement of hemodynamic parameters}

At $0,3,6,9$ and $18 \mathrm{~h}$ after CLP or sham surgery, the carotid artery catheter was connected to a pressure transducer (P23ID, Statham, Oxnard, CA, USA) for the measurement of phasic blood pressure, mean arterial blood pressure (MAP) and heart rate, which were displayed on a polygraph recorder (MacLab/4e, AD Instruments Pty Ltd., Castle Hill, Australia). In addition, after recording of hemodynamic parameters at 0 and $18 \mathrm{~h}$, animals were intravenously given one dose of noradrenaline (NE, $1 \mu \mathrm{g} / \mathrm{kg}$ ) to examine their pressor responses. The vasopressor reactivity was analyzed by integrating the area under the pressure waveform induced by $\mathrm{NE}$ bolus. In order to normalize results of pressor responses to $\mathrm{NE}$ in all groups, we calculated the values of pressor responses to NE at time 0 (baseline) of each group as $100 \%$.

\section{Quantification of organ function and injury}

At 0, 9 and $18 \mathrm{~h}$ after CLP or sham surgery, the arterial blood samples were collected, and then immediately replaced by equal volumes of sterile saline. The total amount of blood removed was about $2.6 \mathrm{~mL}$. Some blood $(160 \mu \mathrm{L})$ was used to analyze the levels of $\mathrm{pH}$, bicarbonate $\left(\mathrm{HCO}_{3}{ }^{-}\right)$, arterial carbon dioxide tension $\left(\mathrm{PaCO}_{2}\right)$, base excess $(\mathrm{BE})$ and potassium concentration by an arterial blood gas analyzer (AVL OPTI Critical Care Analyzer, AVL Scientific Corp., Roswell, GA, USA). In addition, $80 \mu \mathrm{L}$ of plasma was used to analyze the biochemical parameters of liver (alanine aminotransferase (ALT) and aspartate aminotransferase (AST)) and renal (blood urea nitrogen (BUN) and creatinine) functions. In addition, plasma level of lactate dehydrogenase $(\mathrm{LDH})$ was to evaluate the extent of tissue breakdown. All of these biochemical parameters were analyzed by Fuji DRI-CHEM 3030 (Fuji Photo Film Co., Ltd., Tokyo, Japan).

\section{Measurement of plasma interleukin- 6 concentrations}

The plasma samples $(150 \mu \mathrm{L})$ obtained at 0,9 and $18 \mathrm{~h}$ were used. The plasma interleukin-6 (IL-6) was measured in duplicate with an enzyme-linked immunoadsorbent assay kit (R\&D Systems, Inc., Minneapolis, MN, USA) according to the manufacturer's instructions.

\section{Measurement of superoxide production in the plasma, liver and lung}

At the end of experiment (i.e. $18 \mathrm{~h}$ after CLP), the plasma sample was obtained and liver and lung were freshly harvested and cleared of blood and cut into pieces. The $5 \times 5 \mathrm{~mm}$ of lung and liver tissues and $100 \mu \mathrm{L}$ of plasma were transferred to scintillation plates. These scintillation plates containing Krebs' buffer with $1.25 \mathrm{mM}$ lucigenin (final volume of $250 \mu \mathrm{L}$ ) were then placed into a microplate luminometer (Hidex Microplate Luminometer, Turku, Finland) for analysis in duplicate. All tissues of liver and lung were then dried in a $95^{\circ} \mathrm{C}$ oven for $24 \mathrm{~h}$. The results were expressed as counts per sec (in each $\mathrm{mg}$ of liver and lung or in each $100 \mu \mathrm{L}$ of plasma).

\section{Western blot analysis of tissues}

Following protein isolation, equivalent amounts of protein were loaded onto a $10 \%$ (for inducible nitric oxide synthase (iNOS)) and $12 \%$ (for caspase-3) polyacrylamide gel for electrophoresis and blotting. After being blocked for $1 \mathrm{~h}$ at ambient temperature, the membrane was incubated overnight at $4{ }^{\circ} \mathrm{C}$ with polyclonal antimouse iNOS antibody (BD Transduction Laboratories, Lexington, KY, USA) or polyclonal anti-rabbit cleaved caspase 3 antibody (Cell Signaling Technology, Danvers, CO, USA) at a 1: 1000 dilution. The blots were washed and incubated with horseradishperoxidase-coupled secondary antibody (diluted 1:1500; BD Transduction Laboratories, Lexington, KY, USA) for $1 \mathrm{~h}$ at ambient temperature. The blots were then stripped and reprobed with $\beta$-actin antibody (1:3000; BD Transduction Laboratories, Lexington, KY, USA) as internal control. Immunoreactivity was visualized using an enhanced chemiluminescence reaction kit 


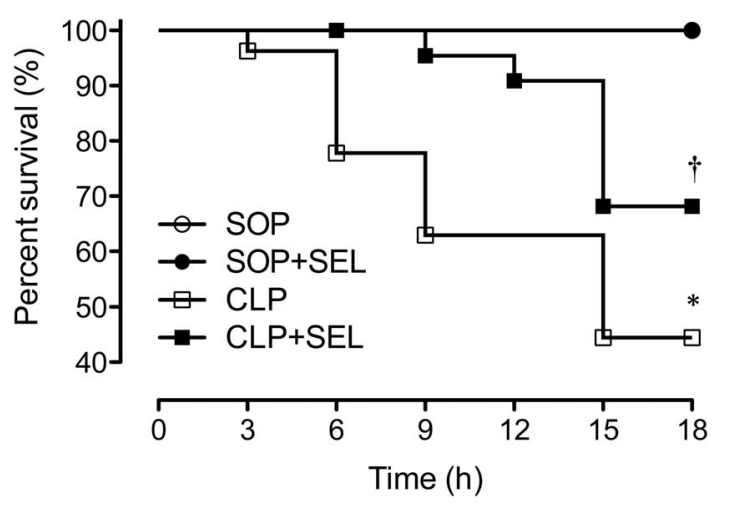

Figure 1. Administration of selegiline (SEL) improves survival after polymicrobial sepsis. A graph of the Kaplan-Meier survival of rats which received sham operation (SOP, $n=11)$, SOP and selegine administration (SOP + SEL, $3 \mathrm{mg} / \mathrm{kg}$, i.v. at $3 \mathrm{~h}$ after SOP, $\mathrm{n}=11$ ), cecal ligation and puncture plus saline $(C L P, n=27)$ and CLP and SEL administration (CLP $+\mathrm{SEL}, 3 \mathrm{mg} / \mathrm{kg}$, i.v. at $3 \mathrm{~h}$ after CLP, $\mathrm{n}=23$ ). The survival time was recorded for 18 hrs. ${ }^{*} P<0.05$, CLP vs. SOP; $\uparrow P<0.05$, with vs. without SEL in animals treated with CLP. Data are expressed as percentage of rats survived at the observed time point.

doi:10.1371/journal.pone.0108455.g001

(Amersham Pharmacia Biotech, Buckinghamshire, UK) and quantified by scanning densitometer.

\section{Histological assessment}

Specimens of liver and lung were harvested for histological analysis as previously described [19]. Briefly, the fixed tissues were dehydrated and embedded in paraffin. Each paraffin block was processed into $4-\mu \mathrm{m}$-thick slices that were stained with hematoxylin and eosin. This histological alteration was quantitatively analyzed as an index of the severity of neutrophil infiltration in 5 animals of each group. The index was determined by counting the numbers of neutrophil in 10 randomly selected high-power fields evaluated by a pathologist in a blinded fashion.

\section{Cell cultures and cell viability assay}

Human aortic endothelial cells (HAECs, Cascade Biologics) were grown in Medium 200 (Cascade Biologics) supplemented with low serum growth supplement (Cascade Biologics) in an atmosphere of $95 \%$ air and $5 \% \mathrm{CO}_{2}$ at $37^{\circ} \mathrm{C}$ in plastic flasks. The final concentrations of the components in Medium 200 contained $2 \%$ fetal bovine serum (Gibco-BRL), $1 \mu \mathrm{g} / \mathrm{mL}$ hydrocortisone, $10 \mathrm{ng} / \mathrm{mL}$ human epidermal growth factor, $3 \mathrm{ng} / \mathrm{mL}$ human fibroblast growth factor, $10 \mu \mathrm{g} / \mathrm{mL}$ heparin, and $1 \%$ antibioticantimycotic mixture (Gibco-BRL). At confluence, the cells were subcultured at a ratio 1:3 and used at passage numbers 3-8. Cell viability was always found to be greater than $95 \%$ by using the trypan blue exclusion method or a 3-(4,5-dimethylthiazol-2- yl)2,5-diphenyl tetrazolium bromide assay (Sigma-Aldrich).

\section{Measurement of reactive oxygen species production}

We determined the effect of SEL on reactive oxygen species (ROS) production in HAECs by fluorometric assay using $2^{\prime}, 7^{\prime}$ dichlorofluorescein diacetate (DCFH-DA, Molecular Probes) as a probe for the presence of $\mathrm{H}_{2} \mathrm{O}_{2}$. HAECs were pre-treated with SEL in 24-well plates for $18 \mathrm{~h}$, and subsequently combined with $25 \mathrm{ng} / \mathrm{mL}$ of lipopolysaccharide (LPS; E. coli serotype 0127:B8, L3127; Sigma-Aldrich) incubation for 3 or $24 \mathrm{~h}$. Cells were subjected to incubation with DCFH-DA $20 \mu \mathrm{M}$ for 45 min after the removal of SEL. Fluorescence intensity (relative fluorescence
A

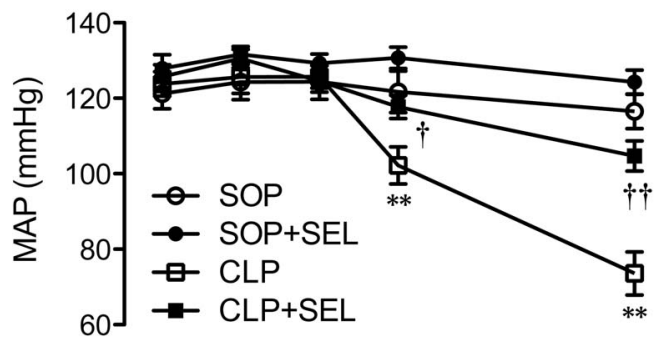

$\mathrm{B}$

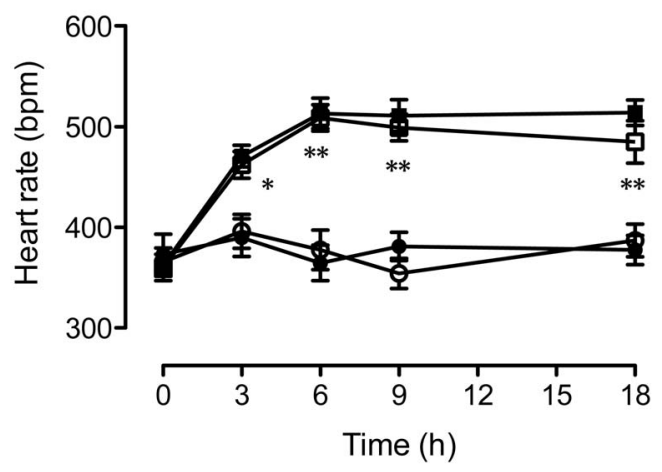

Figure 2. Effects of selegiline on hemodynamics. Alterations in (A) mean arterial pressure (MAP) and (B) heart rate during the experimental period. Rats underwent sham operation (SOP), SOP plus selegiline administration ( $3 \mathrm{mg} / \mathrm{kg}$, i.v., SOP + SEL), cecal ligation and puncture (CLP), or CLP plus SEL administration (3 mg/kg, i.v., CLP + SEL). Data are expressed as mean \pm SEM, $n=10$ in each group for all timepoints. ${ }^{*} p<0.05$ and ${ }^{* *} p<0.01$, CLP vs. SOP; $\dagger p<0.05$ and $\dagger \dagger p<0.01$, with vs. without SEL in animals treated with CLP.

doi:10.1371/journal.pone.0108455.g002

units) was measured at 485-nm excitation and 530-nm emission using a fluorescence micro-plate reader (VICTPR2 Multilabel Readers, USA). Incubation of SEL $(1-100 \mu \mathrm{g} / \mathrm{mL})$ lasting $24 \mathrm{~h}$ had no effect on the cell viability of HAECis.

\section{Western Blotting analysis of HAECs}

Western blot analysis was conducted to determine the changes in expression of LPS-induced iNOS by SEL. Briefly, HAEGs was lysed in a buffer containing $62.5 \mathrm{mM}$ Tris-HCl, 2\% SDS, $10 \%$ glycerol, $0.5 \mathrm{mM}$ PMSF, $2 \mu \mathrm{g} / \mathrm{mL}$ aprotinin, $2 \mu \mathrm{g} / \mathrm{mL}$ pepstatin, and $2 \mu \mathrm{g} / \mathrm{mL}$ leupeptin. The whole-cell lysates were subjected to SDS-polyacrylamide (8\%) gel electrophoresis, followed by electroblotting. Membranes were incubated with monoclonal anti-mouse iNOS antibody (1:1000; BD Transduction Laboratories, Lexington, KY, USA), monoclonal anti-mouse $\beta$-actin antibody (1:10000; Chemicon, Temecula, CA, USA) for overnight, and then incubated for $2 \mathrm{~h}$ with a secondary antibody labeled with horseradish peroxidase. Bands were visualized by chemiluminescence detection reagents (NEN Life Science Products, Boston, MA, USA). Densitometic analysis was conducted with the Image Quant (Promega) software.

\section{Statistical analysis}

The data are presented as mean \pm SEM of $n$ determinations, where $n$ represents the number of animals studied. The distribution of the variables was assessed with a normality test. Data with a normal distribution were analyzed by a one-way analysis of variance (ANOVA) or two-way ANOVA for repeated measures followed, where appropriate, by a Bonferroni correction 

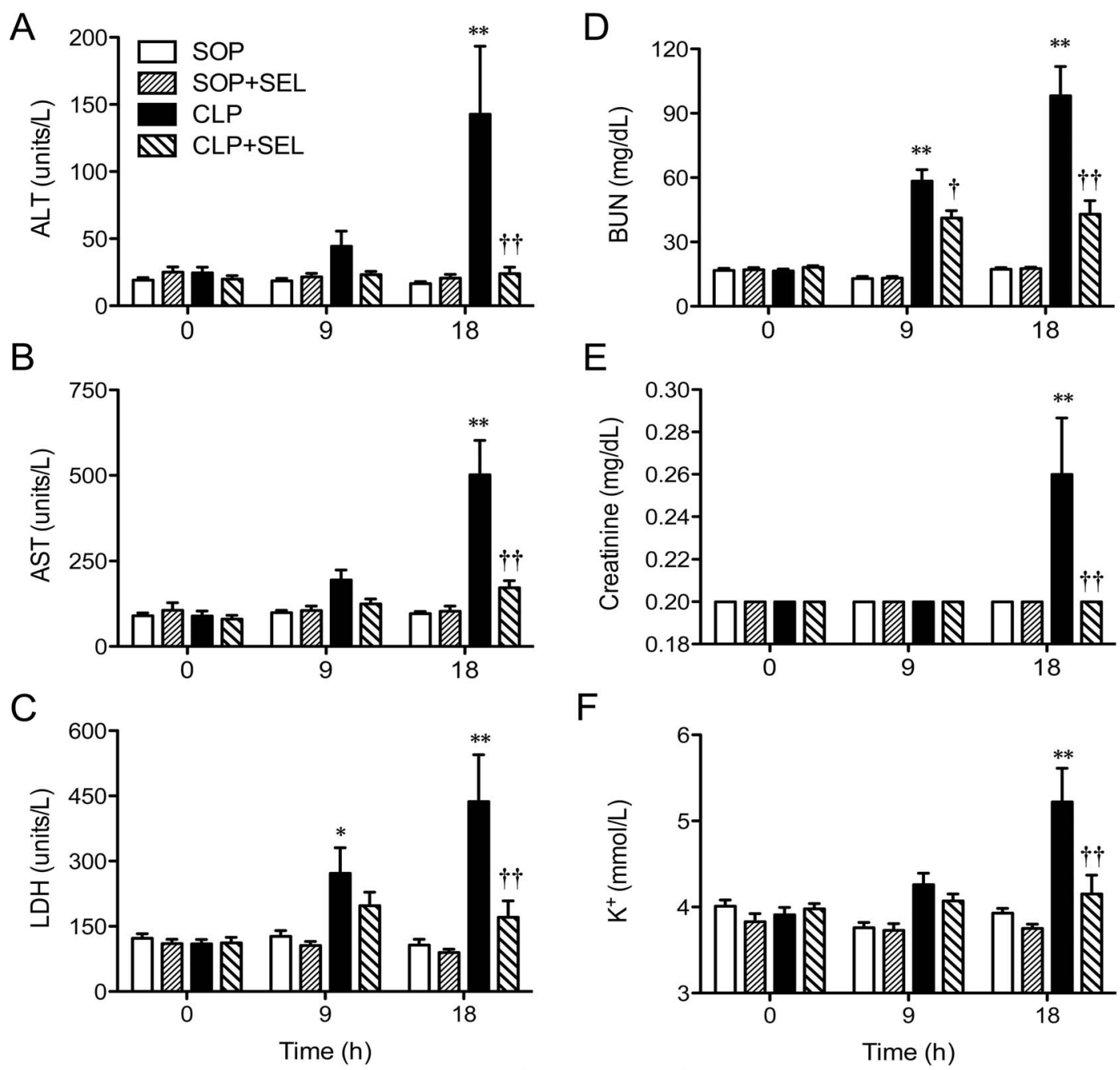

Figure 3. Effects of selegiline on organ function. Alterations in plasma levels of (A) alanine aminotransferase (ALT), (B) asparate aminotransferase (AST), (C) lactate dehydrogenase (LDH), (D) blood urea nitrogen (BUN), (E) creatinine, and (F) blood potassium concentration during the experimental period. Rats underwent sham operation (SOP), SOP plus selegiline administration ( $3 \mathrm{mg} / \mathrm{kg}$, i.v., SOP + SEL), cecal ligation and puncture (CLP), or CLP plus SEL administration ( $3 \mathrm{mg} / \mathrm{kg}$, i.v., CLP $+\mathrm{SEL}$ ). Data are expressed as mean $\pm \mathrm{SEM}, \mathrm{n}=10$ in each group for all time-points. ${ }^{*} p<0.05$ and ${ }^{* *} p<0.01$, CLP vs. SOP; $\uparrow p<0.05$ and $\dagger \dagger p<0.01$, with vs. without SEL in animals treated with CLP.

doi:10.1371/journal.pone.0108455.g003

test. The score for tissue infiltration of neutrophils was compared by the Mann-Whitney $\mathrm{U}$ test. Kaplan-Meier estimates were constructed for overall survival, which was then analyzed by the log-rank test. A $p$ value of less than 0.05 was considered to be statistically significant.

\section{Materials}

Unless otherwise stated all chemicals used were purchased from Sigma-Aldrich.

\section{Results}

\section{Survival rate}

No mortality was observed within $18 \mathrm{~h}$ in all SOP rats $(\mathrm{n}=11$ in each SOP group, figure 1). The survival rate was significantly decreased to $63 \%$ and $44 \%$ (i.e. $17 / 27$ and $12 / 27$ animals) at 9 and $18 \mathrm{~h}$ after CLP, respectively, whereas SEL (3 mg/kg) significantly increased the survival of CLP-treated rats to $96 \%$ and $65 \%$ (i.e., $22 / 23$ and 15/23 animals) at 9 and $18 \mathrm{~h}(p<0.05$, vs. CLP group). However, $1 \mathrm{mg} / \mathrm{kg}$ of SEL did not improve the survival of CLP rats in our preliminary data. Because of clot or kinking of arterial catheter, blood could not be withdrawn for above tests in some of rats, esp. in rats with CLP surgery.

\section{Systemic hemodynamic parameters}

As results shown in Fig. 2, the baseline values for MAP and heart rate among all groups were comparable. The CLP surgery led to a significantly substantial attenuation in MAP at $18 \mathrm{~h}(p<$ 0.01 , vs. SOP group; Fig. 2A), whereas significant and sustained increases in heart rate was observed from $3 \mathrm{~h}$ after CLP $(p<0.05$ or $<0.01$, vs. SOP group; Fig. 2B). The treatment with SEL significantly prevented the severe hypotension at $18 \mathrm{~h}$ after CLP $(p<0.01$, vs. GLP group). However, the CLP-induced tachycardia was not attenuated in rats treated with SEL. The SOP rats treated with saline or SEL exhibited stable hemodynamic conditions during the experimental period and there was no significant 
A
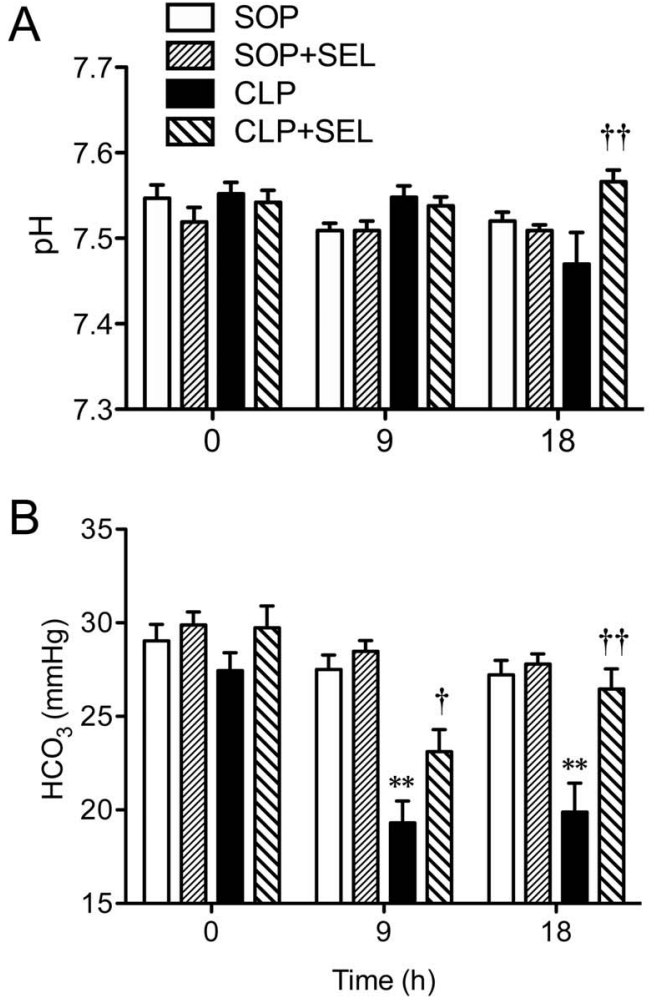

C

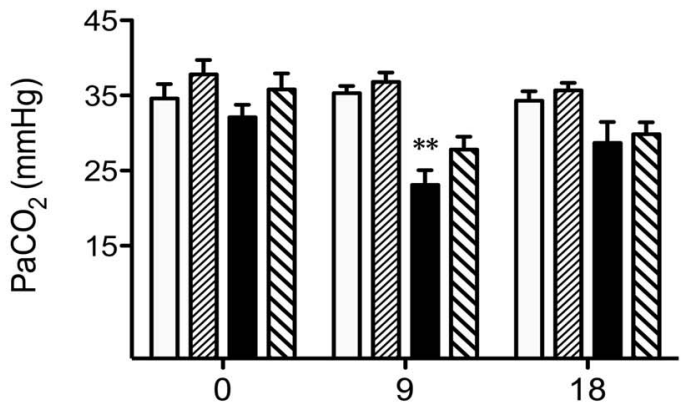

D

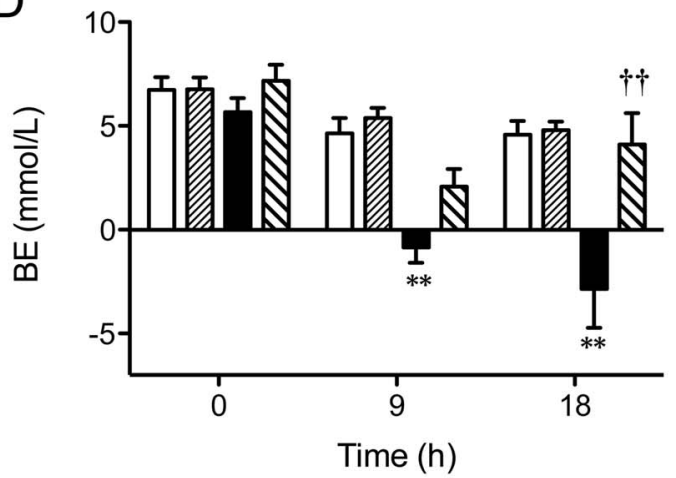

Figure 4. Effects of selegiline on blood potassium concentration and metabolic acidosis. Alterations in arterial blood levels of (A) $\mathrm{pH}$, (B) bicarbonate $\left(\mathrm{HCO}_{3}{ }^{-}\right)$, (C) carbon dioxide tension $\left(\mathrm{PaCO}_{2}\right)$, and $(\mathrm{D})$ base excess (BE) during the experimental period. Rats underwent sham operation (SOP), SOP plus selegiline administration (3 mg/kg, i.v., SOP + SEL), cecal ligation and puncture (CLP), or CLP plus SEL administration (3 mg/kg, i.v., $\mathrm{CLP}+\mathrm{SEL})$. Data are expressed as mean $\pm \mathrm{SEM}, \mathrm{n}=10$ in each group for all time-points. ${ }^{* *} p<0.01, \mathrm{CLP}$ vs. SOP; $\dagger p<0.05$ and $\dagger \dagger p<0.01$, with vs. without SEL in animals treated with CLP.

doi:10.1371/journal.pone.0108455.g004

difference between both groups. In addition, the pressor response to $\mathrm{NE}$ at $18 \mathrm{~h}$ was significantly decreased to $19.5 \pm 2.3 \%$ of baseline in CLP rats $(p<0.01$, vs. SOP group), which was attenuated by SEL $(42.9 \pm 5 \%$ of baseline; $p<0.01$, vs. CLP group). In SOP rats, the pressor response to $\mathrm{NE}$ at $18 \mathrm{~h}$ was $100.4 \pm 7.2 \%$ of baseline.

\section{Biochemical and blood gas parameters}

Baseline values of plasma biochemical and blood gas parameters were not significantly different among all groups. The administration of SEL in the SOP groups had little effect on the biochemical and gas parameters during the experimental period.

The CLP surgery caused time-dependent increases in plasma levels of ALT, AST, LDH, BUN, and creatinine $(p<0.05$ or 0.01 , vs. SOP group; Fig. 3). These increases were significantly attenuated by the treatment of CLP rats with SEL $(p<0.01$, vs. CLP group), indicating that SEL ameliorates liver and kidney injuries induced by CLP. There was no difference in blood potassium concentration in all SOP rats. The rats treated with CLP caused hyperkalemia at $18 \mathrm{~h}(p<0.01$, vs. SOP group; Fig. 3F). However, this hyperkalemia induced by CLP were ameliorated by SEL $(p<0.01$, vs. CLP group).

There was no difference in blood $\mathrm{pH}, \mathrm{HCO}_{3}{ }^{-}, \mathrm{PaCO}_{2}$ and $\mathrm{BE}$ in all SOP rats. The pH level in CLP rats was decreased at $18 \mathrm{~h}$, although it was not significant. However, the rats treated with CLP for $18 \mathrm{~h}$ caused significant decreases of $\mathrm{HCO}_{3}{ }^{-}$and $\mathrm{PaCO}_{2}$ and decreases of $\mathrm{BE}$ at 9 and $18 \mathrm{~h}(p<0.01$, vs. SOP group; Fig. 4).
This indicates that CLP-induced sepsis causes compensated metabolic acidosis. However, this decrease of $\mathrm{HCO}_{3}{ }^{-}$level induced by CLP was ameliorated by SEL at $9 \mathrm{~h}$ and $18 \mathrm{~h}(p<$ 0.05 and $p<0.01$, respectively, vs. CLP group).

Plasma IL- 6 and superoxide levels in the plasma, liver, and lung

In the SOP groups, no significant increase in plasma IL-6 level was observed during the experimental period, indicating that sham surgery and SEL treatment almost have no effect on plasma IL-6 level. The animals received CLP showed a significantly increase in the plasma level of IL-6 at $9 \mathrm{~h}(p<0.01$, vs. SOP group; Fig. 5A), and then declined to near baseline level. However, the treatment of CLP rats with SEL significantly attenuated the increases in plasma IL-6 level at $9 \mathrm{~h}(p<0.01$, vs. CLP group; Fig. 5A).

As results shown in Fig. 5B, animals received CLP had variable increases in the superoxide levels of plasma $(p<0.01)$, liver $(p<$ $0.05)$ and lung $(p<0.01)$, whereas the treatment of CLP rats with SEL significantly attenuated the production of superoxide in these specimens. However, SEL alone had no effect on the changes of these superoxide levels in the SOP group.

\section{iNOS and cleaved caspase- 3 expression in tissues}

The protein expression of iNOS was detectable in liver and lung homogenates obtained from the SOP groups, whereas a significant induction of iNOS protein was observed from the CLP rats $(p<$ 


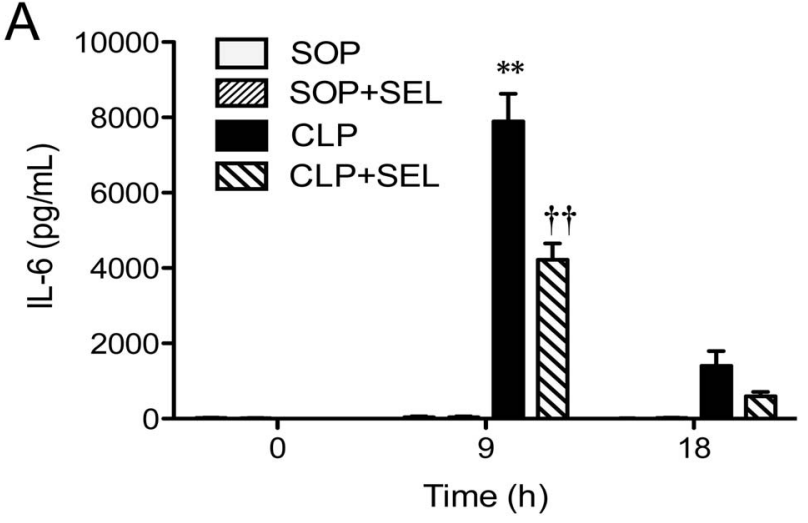

B

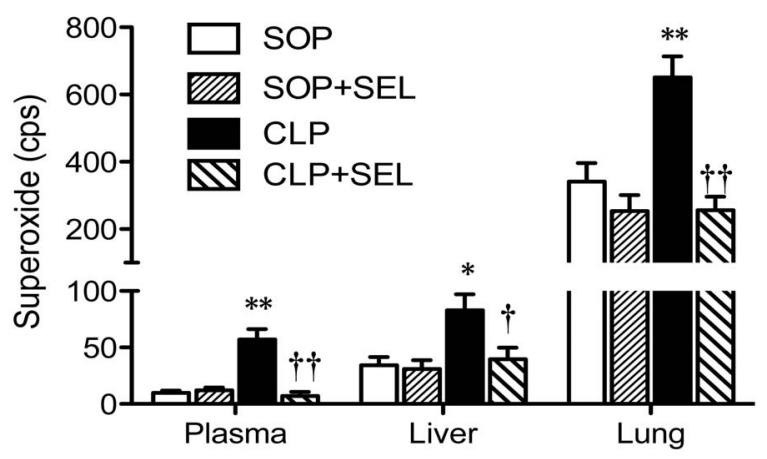

Figure 5. Effects of selegiline on interleukin- 6 and superoxide levels. Alterations in (A) plasma interleukin-6 (IL-6) levels during the experimental period and (B) superoxide levels in plasma, liver and lung at $18 \mathrm{~h}$ after surgery. Rats underwent sham operation (SOP), SOP plus selegiline administration ( $3 \mathrm{mg} / \mathrm{kg}$, i.v., SOP $+\mathrm{SEL})$, cecal ligation and puncture (CLP), or CLP plus SEL administration ( $3 \mathrm{mg} / \mathrm{kg}$, i.v., CLP + SEL). Data are expressed as mean \pm SEM, $n=10$ in each group for all timepoints. ${ }^{*} p<0.05$ and ${ }^{* *} p<0.01$, CLP vs. SOP; $\dagger p<0.05$ and $\dagger \dagger p<0.01$, with vs. without SEL in animals treated with CLP. doi:10.1371/journal.pone.0108455.g005

0.01 in the liver and $p<0.05$ in the lung, vs. SOP group; Fig. 6A). The treatment of CLP rats with SEL significantly reduced the expression of iNOS ( $p<0.05$, vs. CLP group) in liver and lung. The expression of caspase- 3 was significantly higher in liver from the CLP group than from the SOP group $(p<0.01$; Fig. 6B), whereas the treatment of CLP rats with SEL significantly reduced this caspase- 3 expression ( $p<0.01$, vs. CLP group). However, the protein expression of caspase-3 in lung homogenates was not different among all groups (Fig. 6B).

\section{Histopathological changes and neutrophil filtration}

Stained specimens from CLP rats revealed (i) increased interstitial edema and marked necrosis in the liver (Fig. 7), and (ii) increased interstitial edema and decreased alveolar spaces in the lung compared to those from the SOP group (Fig. 8). However, the histopathological changes in these tissues were attenuated after SEL treatment. Light microscopy only showed a little infiltration or sequestration of neutrophil in liver and lung from the SOP group, whereas overt infiltrations of neutrophil in these tissues were observed in CLP rats $(2.8 \pm 0.2$ in liver and $3.2 \pm 0.2$ in lung; $p<0.05)$. However, in CLP rats treated with SEL, the neutrophil infiltrations were significantly reduced $(1.6 \pm 0.4$ in liver and $2 \pm 0.3$ in lung; $p<0.05)$.
A
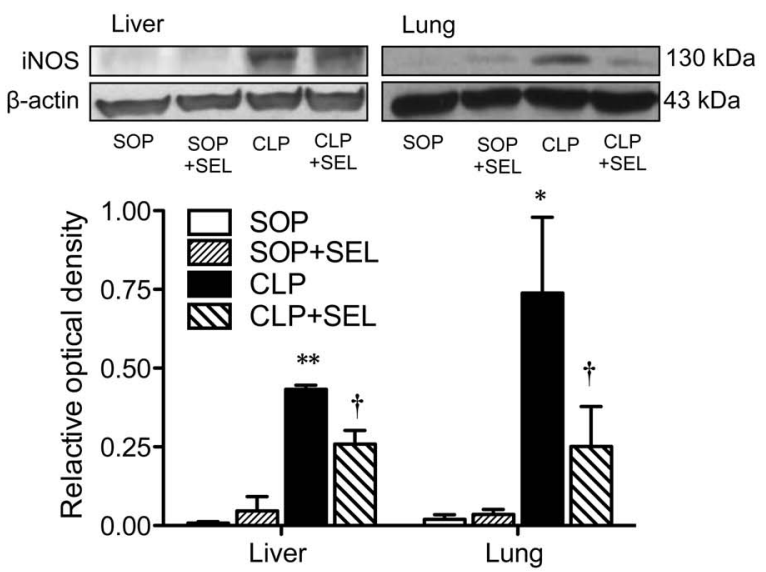

B
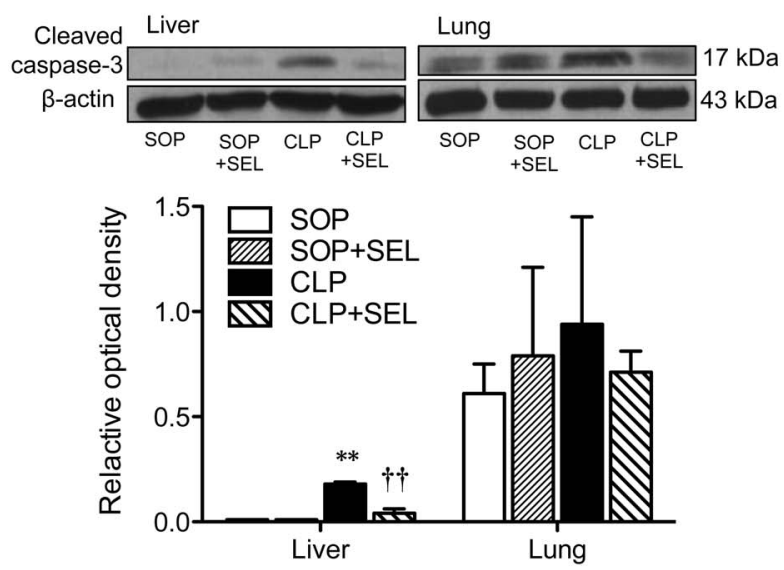

Figure 6. Immunoblot analysis of inducible nitric oxide synthase and cleaved caspase- 3 expression in tissues. Rats underwent sham operation (SOP), SOP plus selegiline administration (3 $\mathrm{mg} / \mathrm{kg}$, i.v., SOP $+\mathrm{SEL}$ ), cecal ligation and puncture (CLP), or CLP plus $\mathrm{SEL}$ administration (3 mg/kg, i.v., CLP + SEL). Liver and lung tissues were harvested at $18 \mathrm{~h}$ after surgery. The summary of quantification of densitometric measurement as ratio of $(A)$ inducible nitric oxide synthase (iNOS) and (B) cleaved caspase-3 relative to $\beta$-actin is presented. Typical Western blots are shown on the upper panel of each figure. $\beta$-actin served as loading control. Data expressed as mean \pm SEM, $\mathrm{n}=3$ in each group. ${ }^{*} p<0.05$ and ${ }^{* *} p<0.01$, CLP vs. SOP; $\dagger p<$ 0.05 and $\dagger \dagger p<0.01$, with vs. without SEL in animals treated with CLP. doi:10.1371/journal.pone.0108455.g006

\section{ROS generation and iNOS expression in LPS-cultured HAECS}

In the present study, intracellular ROS generation was increased by LPS and was unaffected by the pretreatment of SEL $(0.1-10 \mu \mathrm{g} / \mathrm{mL})$ (Figure 9). Western blot analysis also showed that pretreatment of HAECs with SEL $(10 \mu \mathrm{g} / \mathrm{mL})$ significantly increased endothelial iNOS expression (Fig. 10) in the conditioned medium.

\section{Discussion}

In this study, the administration of CLP-induced sepsis rats with SEL (3 mg/kg, i.v.) (i) increased arterial blood pressure and pressor response to NE, (ii) reduced plasma levels of biochemical parameters, (iii) attenuated metabolic acidosis and hyperkalemia, and (iv) ameliorated histopathological changes. This study provides novel evidence that the application of SEL seems to improve survival in the CLP-induced sepsis rats as a consequence 

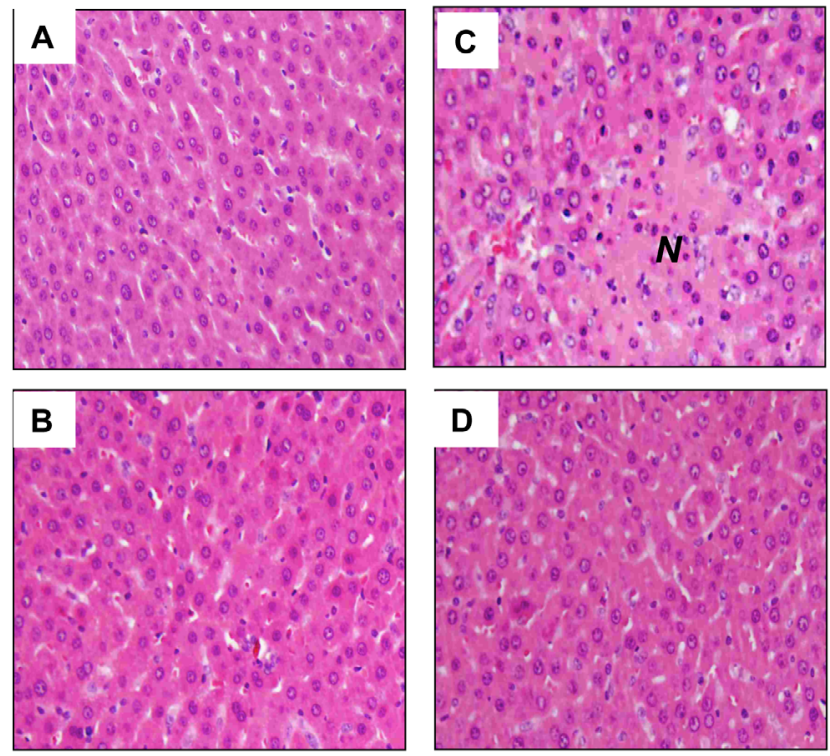

Figure 7. Histological analysis of liver. Liver tissue section stained with hematoxylin and eosin. Rats underwent (A) sham operation (SOP), (B) SOP plus SEL administration (3 mg/kg, i.v., SOP + SEL), (C) cecal ligation and puncture (CLP), or (D) CLP plus SEL administration ( $3 \mathrm{mg} /$ $\mathrm{kg}$, i.v., CLP $+\mathrm{SEL}$ ). Tissues were harvested at $18 \mathrm{~h}$ after surgery. $\mathrm{N}$ indicates necrosis area. Shown are representative micrographs from 5 independent experiments in which the same results were obtained. Each, $400 \times$ (original magnification).

doi:10.1371/journal.pone.0108455.g007

of reduced dysfunction/injury of multiple organs. This could be due to attenuation of IL-6 production and superoxide formation and suppression of iNOS and caspase- 3 expression by SEL in animals with CLP-induced sepsis.

This CLP-induced sepsis model, characterized by a biphasic process, mimics many of the pathophysiologic features of clinically relevant polymicrobial sepsis $[21,22]$. An early phase results from a surge of the unbridled reactive oxygen and nitrogen species and proinflammatory cytokines mediated primarily by neutrophils, macrophages and monocytes, whereas a late phase is marked by a sustained immunosuppressive response induced primarily by apoptosis of immune, epithelial and/or endothelial cells [23-26].

Although multiple reactive oxygen and nitrogen species produced by neutrophils and macrophages for killing invading bacteria in the body, these species can also damage host tissues when they are produced superfluously. Furthermore, together with increased amounts of nitric oxide (NO) that are produced by the iNOS, superoxide forms the highly reactive peroxynitrite that induces irreversible damage to proteins, causing mitochondrial dysfunction and organ failure [27]. Our present study demonstrated that superoxide production and iNOS expression were increased in CLP-induced septic rats, which were attenuated by SEL administration. These observations are consistent with previous studies showing that SEL prevents the increase of oxidative products in microvascular endothelial cells exposed to burn serum [28]. However, in our in vitro study, pretreatment of SEL $(0.1-10 \mu \mathrm{g} / \mathrm{mL})$ did not attenuate intracellular ROS generation and endothelial iNOS expression in HAECs treated with LPS. Similarly, Chakravarti et al. also revealed that $5 \mathrm{nM}$ deprenyl (i.e. SEL) in cultures of RAW 264.7 cells did not affect iNOS expression [29]. Moreover, it has been shown that proinflammatory cytokines can also induce excessive productions of reactive oxygen and nitrogen species [30]. Our in vivo data
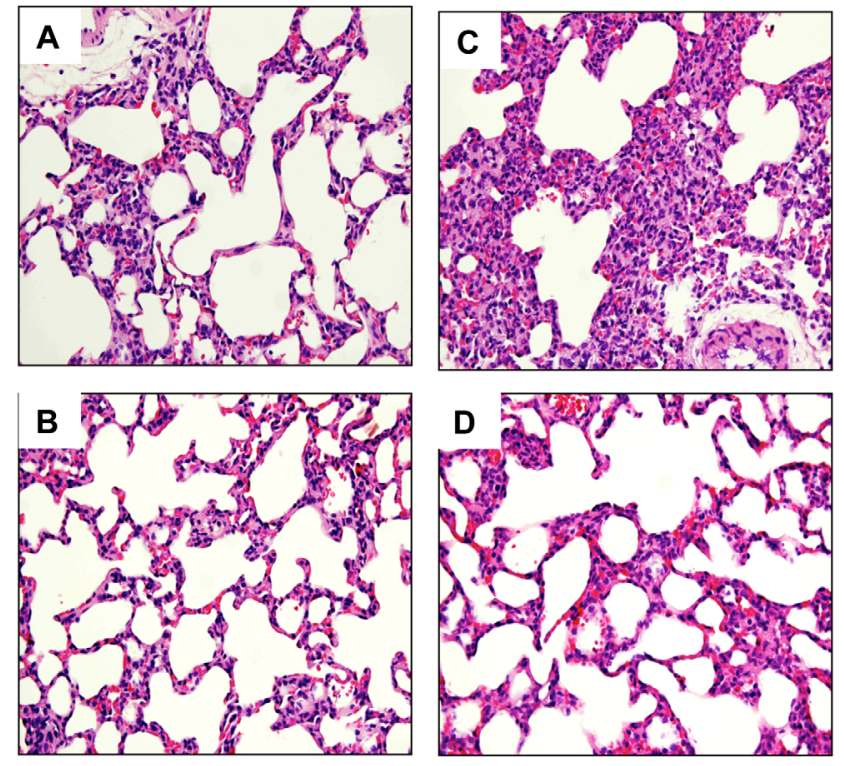

Figure 8. Histological analysis of lung. Lung tissue section stained with hematoxylin and eosin. Rats underwent (A) sham operation (SOP), (B) SOP plus SEL administration (3 $\mathrm{mg} / \mathrm{kg}$, i.v., SOP + SEL), (C) cecal ligation and puncture (CLP), or (D) CLP plus SEL administration (3 mg/ kg, i.v., CLP + SEL). Shown are representative micrographs from 5 independent experiments in which the same results were obtained. Each, $400 \mathrm{X}$ (original magnification).

doi:10.1371/journal.pone.0108455.g008

showed that CLP induced a significant increase of IL-6 in the early septic phase, which was suppressed by SEL. Thus, SEL decreased the inflammatory cytokine levels and the infiltration by neutrophils in organs (e.g. livers and lungs in this study) from sepsis animals. It has been shown that such neutrophil infiltration can lead to vascular dysfunction as well as parenchymal cell injury [31]. Based on these observations, we suggest that SEL prevents organ injury in sepsis most likely by its anti-inflammatory properties.

Indeed, we showed that SEL reduced the increased plasma levels of AST and ALT caused by CLP, which are intracellular

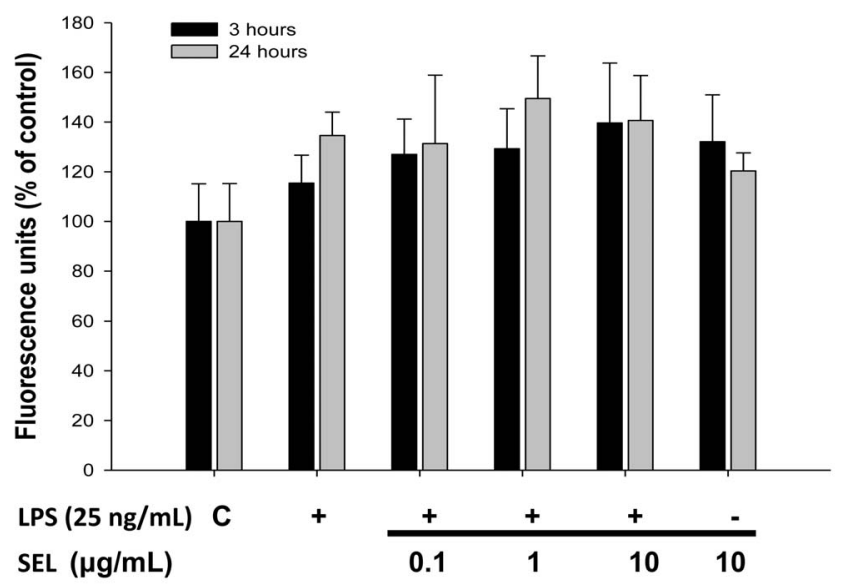

Figure 9. Effects of selegiline on reactive oxygen species in HAECs. Generation of reactive oxygen species induced by lipopolysaccharide (LPS) was unaffected by the treatment of selegiline (SEL) in HAECs. HAECs were pre-incubated with SEL for $18 \mathrm{~h}$, followed by the incubation in $25 \mathrm{ng} / \mathrm{mL}$ of LPS for 3 or $24 \mathrm{~h}$. Data are expressed as mean \pm SEM of three independent experiments. doi:10.1371/journal.pone.0108455.g009 


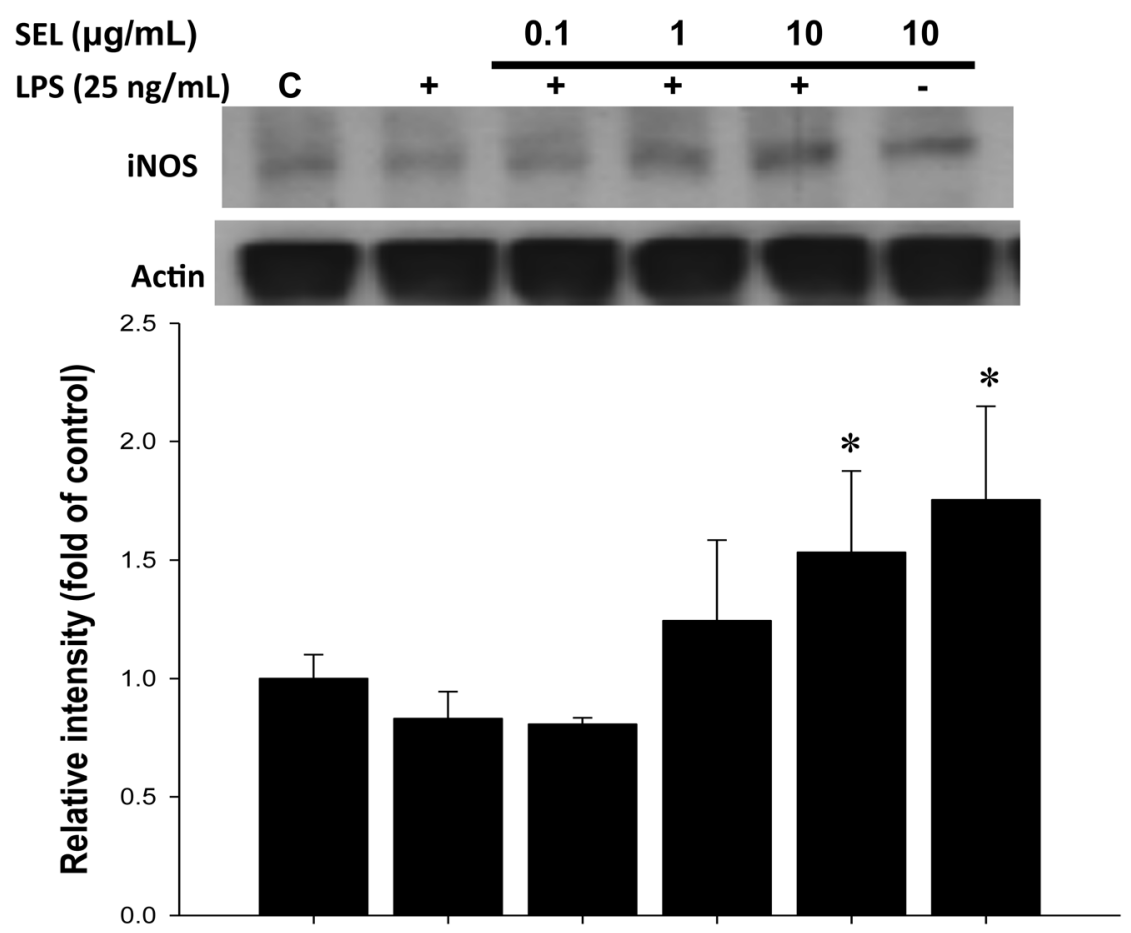

Figure 10. Immunoblot analysis of inducible nitric oxide synthase expression in HAECs. Selegiline (SEL) enhanced the iNOS expression in lipopolysaccharide (LPS)-cultured HAECs. HAECs were pre-treated with SEL for $18 \mathrm{~h}$, and subsequently incubated in $25 \mathrm{ng} / \mathrm{mL}$ of LPS for $24 \mathrm{~h}$. Data are expressed as mean \pm SEM of three independent experiments. ${ }^{*} p<0.05$ compared to LPS alone. doi:10.1371/journal.pone.0108455.g010

components of liver and released into serum during ongoing cell damage. This is consistent with the histological finding showing necrotic cells in the liver from CLP rats. There is increasing evidence that, in addition to cellular necrosis, the apoptotic mode of cell death also plays a pivotal role in the pathogenesis of sepsis syndrome [24,32]. Apoptotic cell death occurs primarily through extrinsic death-receptor pathway and/or intrinsic mitochondria pathway, which can be activated by diverse stimuli, including cytokines and free radicals [33]. The extrinsic or intrinsic pathway can activate caspase-3, leading to the degradation of cellular proteins and the destruction of cell integrity [34]. It has been shown that SEL reduces apoptosis by modulating Bcl-2 and BAX and inhibiting caspase- 3 activity in a number of cell types $[11,16,28]$. Indeed, the decreased of the caspase- 3 protein expression was also observed in the liver of the CLP + SEL group compared to that of the CLP group of animals in our present study. These results suggest that the beneficial effect of SEL on inflammation, tissue injury or apoptosis is further strengthened by the favorable survival outcome in the CLP group. The SELtreated CLP animals had a $21 \%$ survival benefit over CLP controls.

In our study, SEL could attenuated caspase 3 expression in the liver of septic rats at $18 \mathrm{~h}$ after CLP, but not in the lung tissue. However, Tharaken et al. showed that SEL could prevent activation of caspase- 3 in mesenteric vasculature in rats with hemorrhagic shock followed by $60 \mathrm{~min}$ of resuscitation [18]. Therefore, different experimental model, time point, and tissues may result in different effects of SEL on the caspase 3 expression.

It has been shown that amphetamine-like drugs, such as SEL and its major metabolites, L-methamphetamine and L-amphetamine, cause tachycardia [35,36], hypotension [37,38] or hypertension [39]. Allard et al. report that $3 \mathrm{mg} / \mathrm{kg}$ of SEL induces only a transient decrease in blood pressure, which returns to its baseline value after $4 \mathrm{~min}$ [40]. In addition, anorexia/nausea, musculoskeletal injuries, and cardiac arrhythmias occurred more often in patients receiving SEL compared with those receiving placebo [41]. Apart from these adverse effects, increased rates of elevated serum AST and ALT levels were noted [41]. However, $3 \mathrm{mg} / \mathrm{kg}$ of SEL used in this study neither affected MAP and heart rate, nor changed serum AST and ALT levels in sham control rats during the experimental period.

However, the current study has some limitations which need to be addressed. First, only one single intravenous dose of SEL was used, and consequently, we cannot exclude the possibility that multiple doses or continuous infusion could yield better outcome. Second, SEL was given at $3 \mathrm{~h}$ after CLP, nevertheless, the effect of SEL used in the late phase of sepsis is unknown. Third, this experimental sepsis model could not lead to profound hypoxemia at the end of study, indicating that the CLP is not a suitable experimental model of acute lung injury with significant blood gas exchange impairment, including a severe hypoxemic condition.

In conclusion, we used the most clinically relevant sepsis model to monitor sepsis-induced multiple organ dysfunction, and our findings support the hypothesis that SEL improved survival, minimized histological changes and prevented sepsis-induced multiple organ dysfunction by its anti-inflammatory and antiapoptosis properties. This was based on the attenuation of IL-6 and superoxide production as well as the reduction of iNOS and caspase-3 expression in various tissues by SEL in animals with sepsis. Thus, we suggest that SEL could be a potential adjuvant for protecting tissues from oxidative stress and preventing organ dysfunction caused by CLP-induced sepsis. 


\section{Supporting Information}

Checklist S1 The ARRIVE Guidelines Checklist. (PDF)

\section{References}

1. Schlichting D, McCollam JS (2007) Recognizing and managing severe sepsis: a common and deadly threat. South Med J 100: 594-600.

2. Fisher CJ Jr, Agosti JM, Opal SM, Lowry SF, Balk RA, et al. (1996) Treatment of septic shock with the tumor necrosis factor receptor:Fc fusion protein. The Soluble TNF Receptor Sepsis Study Group. N Engl J Med 334: 1697-1702.

3. Hotchkiss RS, Swanson PE, Knudson CM, Chang KC, Cobb JP, et al. (1999) Overexpression of $\mathrm{Bcl}-2$ in transgenic mice decreases apoptosis and improves survival in sepsis. J Immunol 162: 4148-4156.

4. Hotchkiss RS, Chang KC, Swanson PE, Tinsley KW, Hui JJ, et al. (2000) Caspase inhibitors improve survival in sepsis: a critical role of the lymphocyte. Nat Immunol 1: 496-501.

5. Bommhardt U, Chang KC, Swanson PE, Wagner TH, Tinsley KW, et al. (2004) Akt decreases lymphocyte apoptosis and improves survival in sepsis. J Immunol 172: 7583-7591.

6. Hauser RA (2009) New considerations in the medical management of early Parkinson's disease: impact of recent clinical trials on treatment strategy. Parkinsonism Relat Disord 15 Suppl 3: S17-21.

7. Chen JJ (2010) Parkinson's disease: health-related quality of life, economic cost, and implications of early treatment. Am J Manag Care 16 Suppl: S87-93.

8. Mizuno Y, Kondo T, Kuno S, Nomoto M, Yanagisawa N (2010) Early addition of selegiline to L-Dopa treatment is beneficial for patients with Parkinson disease. Clin Neuropharmacol 33: 1-4.

9. Nagatsu T, Sawada M (2006) Molecular mechanism of the relation of monoamine oxidase $\mathrm{B}$ and its inhibitors to Parkinson's disease: possible implications of glial cells. J Neural Transm Suppl 71: 53-65.

10. Naoi M, Maruyama W, Yi H, Inaba K, Akao Y (2009) Mitochondria in neurodegenerative disorders: regulation of the redox state and death signaling leading to neuronal death and survival. J Neural Transm 116: 1371-1381.

11. Tatton W, Chalmers-Redman R, Tatton N (2003) Neuroprotection by deprenyl and other propargylamines: glyceraldehyde-3-phosphate dehydrogenase rather than monoamine oxidase B. J Neural Transm 110: 509-515.

12. Magyar K, Szende B (2004) (-)-Deprenyl, a selective MAO-B inhibitor, with apoptotic and anti-apoptotic properties. Neurotoxicology 25: 233-242.

13. Knollema S, Aukema W, Hom H, Korf J, ter Horst GJ (1995) L-deprenyl reduces brain damage in rats exposed to transient hypoxia-ischemia. Stroke 26: 1883-1887.

14. Simon L, Szilagyi G, Bori Z, Orbay P, Nagy Z (2001) (-)-D-Deprenyl attenuates apoptosis in experimental brain ischaemia. Eur J Pharmacol 430: 235-241.

15. Sivenius J, Sarasoja T, Aaltonen H, Heinonen E, Kilkku O, et al. (2001) Selegiline treatment facilitates recovery after stroke. Neurorehabil. Neural Repair 15: 183-190.

16. Oin F, Shite J, Mao W, Liang CS (2003) Selegiline attenuates cardiac oxidative stress and apoptosis in heart failure: association with improvement of cardiac function. Eur J Pharmacol 461: 149-158.

17. Kiray M, Ergur BU, Bagriyanik A, Pekcetin C, Aksu I, et al. (2007) Suppression of apoptosis and oxidative stress by deprenyl and estradiol in aged rat liver. Acta Histochem 109: 480-485.

18. Tharakan B, Whaley JG, Hunter FA, Smythe WR, Childs EW (2010) (-)Deprenyl inhibits vascular hyperpermeability after hemorrhagic shock. Shock. 33: $56-63$.

19. Tsao CM, Chen SJ, Shih MC, Lue WM, Tsou MY, et al. (2010) Effects of terbutaline on circulatory failure and organ dysfunction induced by peritonitis in rats. Intensive Care Med 36: 1571-1578.

20. Nemzek JA, Xiao HY, Minard AE, Bolgos GL, Remick DG (2004) Humane endpoints in shock research. Shock 21: 17-25.

\section{Author Contributions}

Conceived and designed the experiments: CMT HCH CCW. Performed the experiments: JGJ SJC. Analyzed the data: CMT SJC WJL HCH GCW. Contributed reagents/materials/analysis tools: SMK TCW. Wrote the paper: GMT GCW.

21. Oberholzer A, Oberholzer C, Moldawer LL (2001a) Sepsis syndromes: understanding the role of innate and acquired immunity. Shock 16: 83-96.

22. Yang S, Chung CS, Avala A, Chaudry IH, Wang P (2002) Differential alterations in cardiovascular responses during the progression of polymicrobial sepsis in the mouse. Shock 17: 55-60.

23. Oberholzer C, Oberholzer A, Clare-Salzler M, Moldawer LL (2001b) Apoptosis in sepsis: a new target for therapeutic exploration. FASEB J 15: 879-892.

24. Hattori Y, Takano K, Teramae H, Yamamoto S, Yokoo H, et al. (2010) Insights into sepsis therapeutic design based on the apoptotic death pathway. J Pharmacol Sci 114: 354-365.

25. Olguner CG, Koca U, Altekin E, Ergür BU, Duru S, et al. (2013) Ischemic preconditioning attenuates lipid peroxidation and apoptosis in the cecal ligation and puncture model of sepsis. Exp Ther Med 5: 1581-1588.

26. Tsai KL, Liang HJ, Yang ZD, Lue SI, Yang SL, et al. (2014) Early inactivation of PKCE associates with late mitochondrial translocation of Bad and apoptosis in ventricle of septic rat. J Surg Res 186: 278-286.

27. Alvarez S, Evelson PA (2007) Nitric oxide and oxygen metabolism in inflammatory conditions: sepsis and exposition to polluted ambients. Front Biosci 12: 964-974.

28. Whaley JG, Tharakan B, Smith B, Hunter FA, Childs EW (2009) (-)-Deprenyl inhibits thermal injury-induced apoptotic signaling and hyperpermeability in microvascular endothelial cells. J Burn Care Res 30: 1018-1027.

29. Chakravarti R, Aulak KS, Fox PL, Stuehr DJ (2010) GAPDH regulates cellular heme insertion into inducible nitric oxide synthase. Proc Natl Acad Sci USA 107: 18004-18009.

30. Morgan MJ, Liu ZG (2011) Crosstalk of reactive oxygen species and NF-kappaB signaling. Cell Res 21: 103-115.

31. Jaeschke H, Hasegawa T (2006) Role of neutrophils in acute inflammatory liver injury. Liver Int 26: 912-919.

32. Hotchkiss RS, Nicholson DW (2006) Apoptosis and caspases regulate death and inflammation in sepsis. Nat Rev Immunol 6: 813-822.

33. Wesche-Soldato DE, Swan RZ, Chung CS, Ayala A (2007) The apoptotic pathway as a therapeutic target in sepsis. Curr Drug Targets 8: 493-500.

34. Boatright KM, Salvesen GS (2003) Mechanisms of caspase activation. Curr Opin Cell Biol 15: 725-731.

35. Reynolds GP, Elsworth JD, Blau K, Sandler M, Lees AJ, et al. (1978) Deprenyl is metabolised to methamphetamine and amphetamine in man. Br J Clin Pharmacol 6: 542-544.

36. Glezer S, Finberg JP (2003) Pharmacological comparison between the actions of methamphetamine and 1-aminoindan stereoisomers on sympathetic nervous function in rat vas deferens. Eur J Pharmacol 472: 173-177.

37. Abassi ZA, Binah O, Youdim MB (2004) Cardiovascular activity of rasagiline, a selective and potent inhibitor of mitochondrial monoamine oxidase $\mathrm{B}$ : comparison with selegiline. Br J Pharmacol 143: 371-378.

38. Finberg JP, Gross A, Bar-Am O, Friedman R, Loboda Y, et al. (2006) Cardiovascular responses to combined treatment with selective monoamine oxidase type B inhibitors and L-DOPA in the rat. Br J Pharmacol 149: 647-656.

39. Bexis S, Docherty JR (2006) Effects of MDMA, MDA and MDEA on blood pressure, heart rate, locomotor activity and body temperature in the rat involve alpha-adrenoceptors. Br J Pharmacol 147: 926-934.

40. Allard J, Bernabe J, Derdinger F, Alexandre L, McKenna K, et al. (2002) Selegiline enhances erectile activity induced by dopamine injection in the paraventricular nucleus of the hypothalamus in anesthetised rats. Int J Impot Res 14: 518-522.

41. Yamada M, Yasuhara H (2004) Clinical pharmacology of MAO inhibitors: safety and future. Neurotoxicology 25: 215-21. 